\title{
Risco de suicídio em pacientes em hemodiálise: evolução e mortalidade em três anos
}

\author{
Risk of suicide in hemodyalisis patient: evolution \\ and mortality in three years
}

Jose A. Moura Junior', Carlos A. Marcilio de Souza', Irismar Reis de Oliveira², Roberta Oliveira Miranda², Carlos Teles 3 , José A. Moura Neto ${ }^{4}$

\section{RESUMO}

A morte por suicídio em pacientes portadores de insuficiência renal crônica (IRC) em diálise tem sido reportada há décadas. No Brasil, raros são os estudos que têm mensurado sua prevalência, evolução e mortalidade. Objetivo: Identificar a presença de risco de suicídio, em duas unidades de diálise e analisar a evolução e a mortalidade por três anos. Método: O MINI foi utilizado em três etapas. Foram obtidas as freqüências do transtorno e sua evolução foi acompanhada. A curva de Kaplan-Meier e a regressão de Cox foram aplicadas para estudar a mortalidade. Resultados: Participaram 244 pacientes na primeira etapa, 200, na segunda etapa e 110, na terceira etapa. O risco de suicídio foi diagnosticado em 40 pacientes na primeira etapa, 49, na segunda etapa e sete na terceira etapa. Da primeira para a segunda etapa, nove pacientes morreram, 29 continuaram e 20 outros pacientes passaram a apresentar a condição. Da segunda para a terceira etapa, 13

\section{Palavras-chave}

Risco de suicídio, hemodiálise, MINI International

Neuropsychiatric Interview.

\section{Key-words}

Psychiatric disease,

hemodialysis,

MINI International

Neuropsychiatric Interview. deles morreram, sete continuaram a apresentar e 29 evoluíram para outro transtorno. A incidência de óbitos naqueles sem o transtorno foi de 3,35 e naqueles com risco de suicídio, 9,91 ( $R R=2,87$; IC 95\% [1,69-4,87]). Conclusões: O risco de suicídio teve alta prevalência, e a mortalidade associada a esta condição é elevada.

\section{ABSTRACT}

Risk of suicide is associated with high rates of death in chronic hemodialysis patients. In Brazil only few studies have measured your prevalence, evolution and mortality. Objective: Study the prevalence, evolution and mortality of risk of suicide in two nephrology units for three years. Methods: The Mini was used in three moments. Frequency and evolution of Risk of Suicide was analyzed. KaplanMeier Curve and Cox Regression was used to study the mortality. Results: 244 patients in ${ }^{\text {st }}$ step, 200 in $2^{\text {nd }}$ and 110 in $3^{\text {rd }}$. Risk of suicide was diagnosticated in 40 in $1^{\text {st }}, 49$ in $2^{\text {nd }}$ and seven in $3^{\text {rd }}$ period. Between the $1^{15 t}$ and $2^{\text {nd }}$ period, nine patients death, 29 followed with the condition and 20 others patients presented risk of suicide. Between the $2^{\text {nd }}$ and $3^{\text {rd }}$ period thirteen death, seven followed with the condition and 29 changed the disorder. The death incidence in patients without disorder was 3.35 and in patients with risk was, 9.91 (RR $=2.87$; IC de 95\% [1.69-4.87]). Conclusions: The prevalence of risk of suicide was high, and this condition was associated with high rates of mortality.

1 Fundação para o Desenvolvimento das Ciências, Brotas, Salvador - BA.

2 Universidade Federal da Bahia (UFBA)

Recebido em 


\section{INTRODUÇÃO}

A insuficiência renal crônica (IRC) é uma condição de enorme impacto psicossocial e constitui problema de saúde pública de grande magnitude, inclusive pela sua alta prevalência. O número estimado de pacientes em programa de diálise em todo o mundo é de aproximadamente 1.200.000, número este que aumenta em média 7\% ao ano'. No Brasil, dados da Sociedade Brasileira de Nefrologia (SBN) de 2006 apontam a existência de mais de 70 mil pacientes em terapia substitutiva ${ }^{2}$. O estado da Bahia, por possuir reduzido número de centros de diálise, comparativamente com outros estados, apresenta demanda reprimida de pacientes por diálise. O contingente atual é de aproximadamente 3 mil pacientes em tratamento 3 .

A história natural da IRC modificou-se radicalmente nas últimas décadas com a introdução de métodos de diálise e o desenvolvimento do transplante, o que contribuiu para melhorar substancialmente o prognóstico ${ }^{4}$. É notória a redução da mortalidade e a elevação da expectativa de vida dos pacientes no âmbito das unidades de diálise. No Brasil, dados do censo de 2006 reportam sobrevida ao final de dois anos de $70 \%$ e mortalidade anual de 16,1\%². Em países europeus, a sobrevida estimada de cinco anos entre pacientes dialisados é de $60 \% \%^{5}$. Na Bahia, a mortalidade se situa ao redor de $10 \%$ ao ano, de acordo com os dados da Comissão de Nefrologia da Bahia, de $2000^{3}$.

A IRC demanda que o indivíduo e a sua família se adaptem a um novo padrão de vida. Os pacientes em diálise são submetidos a inúmeras pressões psicológicas e limitações na qualidade de vida que incluem: a) dependência imposta pelo tratamento; b) medo da morte; c) severas restrições impostas pelo regime de diálise; d) complicações físicas da doença; e) perdas impostas pela patologia; f) mudanças na imagem corporal ${ }^{6,7}$. As pressões psicológicas e as limitações de qualidade de vida dos pacientes em hemodiálise estão associadas à maior prevalência e incidência de transtornos psiquiátricos ${ }^{8,9}$.

Entre os transtornos psiquiátricos, a depressão e a ansiedade são as síndromes mais prevalentes, com taxas que variam de $12 \%$ a $45 \%$, a depender da metodologia utilizada, e são descritas como causas independentes de mortalidade $4,8,10$.

A mais grave complicação da depressão é o suicídio. Mortalidade por esta razão em pacientes com IRC tem sido reportada há décadas, tanto naqueles em diálise quanto no pós-transplante renal ${ }^{10-12}$. Estes indivíduos se deparam freqüentemente com oportunidades de efetivar a intenção de suicidar-se, seja faltando às sessões, não tomando corretamente as medicações ou não seguindo as orientações que dizem respeito à dieta e às restrições hídricas.
A psicopatologia é de extrema importância na gênese de numerosos casos de suicídio e, segundo alguns autores, em até $90 \%$ deles, há um diagnóstico psiquiátrico presente ${ }^{13}$. Em hospitais psiquiátricos, a mortalidade por suicídio é marcadamente aumentada, cerca de três a 12 vezes maior que a da população geral, e vem crescendo nos últimos anos ${ }^{14}$.

Em 1990, mais de 1,4 milhão de pessoas se suicidou, o equivalente a, aproximadamente, $1,6 \%$ da mortalidade mundial, ficando o suicídio entre as dez principais causas de morte ${ }^{15}$. Se as tentativas também forem computadas, esse número torna-se de 10 a 20 vezes maior ${ }^{16}$. No Brasil, apesar da pouca confiabilidade dos dados e dos parcos recursos destinados à sistematização de boa coleta, a Fundação Nacional de Saúde computou 6.594 suicídios, em 1995, sendo a maior taxa entre a população de 20 a 39 anos $^{17}$.

As estimativas de suicídio em pacientes em terapia dialítica variam amplamente, na medida em que se considere, ou não, o comportamento de não-adesão à dieta e às medicações, falta às sessões e abandono de tratamento, como tentativa de suicídio. Se assim consideradas, as taxas podem ser tão altas quanto 400 vezes a da população geral (10 suicídios por 100.000 habitantes, em média) ${ }^{11}$. Segundo Mello Jorge, o suicídio é a mais passível de subestimação entre as causas externas ${ }^{18}$. Uma vez caracterizada e diagnosticada, esta condição requer tratamento, o qual está disponível e apresenta nível aceitável de efetividade e segurança ${ }^{19}$. No Brasil, raros são os estudos que têm mensurado a real prevalência desta condição no âmbito das unidades de diálise e falham em acompanhar sua evolução e em determinar sua mortalidade.

Pretendeu-se neste estudo, inicialmente identificar a presença de risco de suicídio, em duas unidades de diálise, acompanhar estes pacientes durante três anos, avaliar a evolução e analisar a mortalidade relacionada à presença e à ausência deste transtorno.

Foi utilizado em três oportunidades, ao longo deste tempo, o MINI Internacional Neuropsychiatric Interview, validado quando em comparação com o Structured Clinical Interview for DSM-III-R, Patient Version (SCID-P) e com o Composite International Diagnostic Interview (CIDI), dois dos mais utilizados instrumentos de diagnóstico da pesquisa psiquiátrica ${ }^{20}$. Por ser um questionário de ampla utilização entre os psiquiatras, é capaz de reconhecer substancial parcela de indivíduos com alterações de saúde mental. A entrevista possui módulo para diagnóstico de risco de suicídio que utiliza como base seis indagações, das quais se espera respostas do tipo "sim" ou "não", e que gradua o risco em "leve", "moderado" ou "elevado". Breve e simples na sua aplicação, pode ser útil na identificação desta condição neste grupo específico de pacientes ${ }^{21}$. Como ponto de partida, foi utilizado o trabalho de Moura, Marcilio de Souza e Oliveira so- 
bre prevalência de transtornos psiquiátricos em pacientes em hemodiálise publicado em $2006^{22}$.

\section{MÉTODOS}

Um estudo longitudinal pelo período de três anos foi realizado em duas clínicas de nefrologia no estado da Bahia: uma em Salvador e a outra em Feira de Santana, maior cidade do interior baiano, distante $108 \mathrm{~km}$ da capital. A amostra inicial foi constituída por 244 pacientes que dializavam nas clínicas e que foram escolhidos de forma randomizada. Todos assinaram o termo de consentimento livre e esclarecido. O protocolo do estudo foi submetido e julgado aprovado pelo Comitê de Ética e Pesquisa do Centro de Pesquisas Gonçalo Moniz (CPqGM) da Fundação Osvaldo Cruz (Fiocruz).

O período do estudo compreendeu três etapas, e em cada uma delas, o MINI foi aplicado: primeira etapa - de outubro de 2001 a fevereiro de 2002, a amostra foi constituída por 244 pacientes; segunda etapa - de fevereiro de 2003 a outubro de 2003, foram estudados 200 pacientes, excluídos aqueles que foram transferidos, transplantados e os que faleceram; terceira etapa - de outubro de 2004 a fevereiro de 2005, a amostra se constituiu de 110 pacientes, também excluídos aqueles que foram transferidos, transplantados ou foram a óbito.

Os pacientes realizavam hemodiálise regular nas clínicas e os critérios de inclusão foram: ser portador de IRC terminal e estar em programa de hemodiálise nas unidades do estudo há mais de 30 dias, ter concordado em participar do estudo e assinado o termo de consentimento livre e esclarecido e encontrar-se em condições clínicas estáveis, não tendo sido internado nos últimos 15 dias. As entrevistas foram realizadas na primeira etapa por uma psiquiatra treinada na aplicação do questionário e nas etapas subseqüentes por uma psicóloga treinada pela primeira profissional.

$\mathrm{Na}$ análise univariada foram obtidas as freqüências absolutas e percentuais dos tipos de transtornos e das variáveis sociodemográficas e clínicas. Posteriormente, na análise bivariada verificou-se a associação e a magnitude desta associação entre o risco de suicídio e as variáveis, mediante o teste qui-quadrado e razões de risco, com seus respectivos intervalos de confiança de $95 \%$.

A evolução do risco de suicídio foi apresentada graficamente quanto a sua distribuição nas três etapas. Verificou-se a freqüência de mudança (evolução) do transtorno entre a primeira e a segunda etapas e entre a segunda e a terceira etapas do estudo, segundo a aplicação do teste qui-quadrado de McNemar. Para análise da mortalidade foram obtidos o número de óbitos e a prevalência de óbito registrada durante o estudo. A curva de Kaplan-Meier foi aplicada para comparar a mortalidade, ao final dos três anos, entre aqueles pacientes que apresentavam risco de suicídio e aqueles que não apresentaram nenhum transtorno no início do estudo.

A análise multivariada foi realizada mediante o modelo de regressão logística não condicional (ou regressão de Cox), obtendo-se a medida de risco OR (ou HR) ajustadas, com seu respectivo intervalo de confiança de $95 \%$. O nível de significância adotado para os testes estatísticos foi de 5\% $(p<0,05)$. Para a construção do banco de dados e as análises necessárias, foram utilizados os programas Epi-Info v 6.4 e o SPSS Windows v.9.0. A correção dos dados foi realizada por meio da análise de consistência e da busca de erros de registro, que foram retificados, quando encontrados, por recuperação dos questionários.

\section{RESULTADOS}

O estudo transcorreu em três etapas, com a aplicação do questionário em cada uma delas. Dos 244 pacientes submetidos ao MINI ao início do estudo, 200 foram analisados na segunda etapa e apenas 110 completaram as três etapas. As causas desta diminuição foram óbitos (32 pacientes antes da segunda etapa e 29 antes da terceira etapa), transferências de Centro, o que impossibilitou a aplicação do teste (12 pacientes antes da segunda etapa e 58 antes da terceira etapa), e, finalmente, algumas poucas recusas de responder ao questionário (três) que ocorreram na terceira etapa.

A idade média foi de 44,5 anos $\pm 15,2$ na primeira etapa, 45,35 anos $\pm 14,2$ na segunda e 46,2 anos $\pm 12,1$ anos na terceira e última. Os percentuais de homens nas etapas foram 58,6\%,60\% e 63,1\%, respectivamente. A Tabela 1 resume as variáveis sociodemográficas e clínicas do estudo nas três etapas.

A prevalência de pelo menos um transtorno psiquiátriCo das 16 condições analisadas pelo MINI foi de 37,3\% na primeira etapa, 57,5\% na segunda etapa e 38,2\% na terceira e última etapa. O Gráfico 1 apresenta estas proporções em cada uma das etapas. O risco de suicídio foi uma condição comum nas duas etapas iniciais do estudo, com 40 pacientes representando $16,4 \%$ da amostra de 244 na primeira etapa, e 49 pacientes representando 24,5\% dos 200 pacientes da segunda etapa. Na terceira e última etapa, o número de pacientes com este transtorno psiquiátrico foi de sete, o que representou 6,4\% da amostra de 110 pacientes. Na classificação por gravidade oferecida pelo questionário, apenas um paciente na primeira etapa e um na segunda tiveram risco elevado. Risco moderado foi diagnosticado em cinco pacientes na primeira etapa e em um na segunda. Todos os outros pacientes tiveram diagnóstico de risco leve. 
Tabela 1. Variáveis sociodemográficas e clínicas dos pacientes em programa de hemodiálise nas três etapas

\begin{tabular}{|c|c|c|c|c|c|c|c|c|c|}
\hline \multirow[t]{2}{*}{ Variáveis } & \multicolumn{3}{|c|}{ 1a Etapa } & \multicolumn{3}{|c|}{ 2a Etapa } & \multicolumn{3}{|c|}{ 3a Etapa } \\
\hline & $n$ & $\%$ & IC $95 \%$ & $n$ & $\%$ & IC $95 \%$ & $n$ & $\%$ & IC $95 \%$ \\
\hline \multicolumn{10}{|l|}{ Demográficas } \\
\hline \multicolumn{10}{|l|}{ Sexo } \\
\hline Masculino & 143 & $58,6 \%$ & {$[52,2-64,8]$} & 120 & $60 \%$ & {$[51,2 \%-68,8 \%]$} & 63 & $56 \%$ & {$[43,7 \%-68,3 \%]$} \\
\hline Feminino & 101 & $41,4 \%$ & {$[35,2-47,9]$} & 80 & $40 \%$ & {$[29,3 \%-50,7 \%]$} & 50 & $44 \%$ & {$[30,2 \%-57,8 \%]$} \\
\hline \multicolumn{10}{|l|}{ Faixa etária } \\
\hline$\leq 20$ anos & 12 & $4,9 \%$ & {$[2,6-8,4]$} & 5 & $2,5 \%$ & {$[-11,2 \%-6,2 \%]$} & 2 & $1,8 \%$ & {$[-16,6 \%-20,2 \%]$} \\
\hline 21 a 40 anos & 97 & $39,8 \%$ & {$[33,6-46,2]$} & 78 & $39 \%$ & {$[28,2 \%-49,8 \%]$} & 45 & $40 \%$ & {$[25,7 \%-54,3 \%]$} \\
\hline 41 a 60 anos & 89 & $36,5 \%$ & {$[30,4-42,9]$} & 80 & $40 \%$ & {$[29,3 \%-50,7 \%]$} & 50 & $44,2 \%$ & {$[30,4 \%-58,0 \%]$} \\
\hline$\geq 61$ anos & 44 & $18,0 \%$ & {$[13,4-23,4]$} & 37 & $18 \%$ & {$[5,6 \%-30,4 \%]$} & 16 & $15 \%$ & {$[-2,5 \%-32,5 \%]$} \\
\hline \multicolumn{10}{|l|}{ Estado civil } \\
\hline Casado & 121 & $49,6 \%$ & {$[43,2-56,0]$} & 96 & $48 \%$ & {$[38,0 \%-58,0 \%]$} & 53 & $47 \%$ & {$[33,6 \%-60,4 \%]$} \\
\hline Solteiro & 102 & $41,8 \%$ & {$[35,5-48,3]$} & 86 & $43 \%$ & {$[32,5 \%-53,5 \%]$} & 52 & $46 \%$ & {$[32,5 \%-59,5 \%]$} \\
\hline Viúvo & 10 & $4,1 \%$ & {$[2,0-7,4]$} & 8 & $4,0 \%$ & {$[-9,6 \%-17,6 \%]$} & 5 & $4,4 \%$ & {$[-13,6 \%-22,4 \%]$} \\
\hline Separado & 11 & $4,5 \%$ & {$[2,3-7,9]$} & 10 & $5,0 \%$ & {$[-8,5 \%-18,5 \%]$} & 3 & $2,6 \%$ & {$[-15,4 \%-20,6 \%]$} \\
\hline \multicolumn{10}{|l|}{ Clínicas } \\
\hline \multicolumn{10}{|l|}{ Tempo em diálise } \\
\hline$\leq 12$ meses & 44 & $18,0 \%$ & {$[13,4-23,4]$} & 0 & $0 \%$ & & 0 & $0 \%$ & {$[0 \%]$} \\
\hline 13 a 24 meses & 49 & $20,1 \%$ & {$[15,2-25,7]$} & 24 & $12 \%$ & {$[-1,0 \%-25,0 \%]$} & 1 & 0,9 & {$[0 \%]$} \\
\hline 25 a 36 meses & 29 & $11,9 \%$ & {$[8,1-16,6]$} & 31 & $15,5 \%$ & {$[2,8 \%-28,2 \%]$} & 3 & $2,7 \%$ & {$[-15,6 \%-21,0 \%]$} \\
\hline 37 a 48 meses & 31 & $12,7 \%$ & {$[8,8-17,5]$} & 31 & $15,5 \%$ & {$[2,8 \%-28,2 \%]$} & 22 & $20,1 \%$ & {$[3,4 \%-36,8 \%]$} \\
\hline 49 a 60 meses & 34 & $13,9 \%$ & {$[9,8-18,8]$} & 29 & $14,5 \%$ & {$[1,7 \%-27,3 \%]$} & 24 & $21,3 \%$ & {$[4,9 \%-37,7 \%]$} \\
\hline$\geq 61$ meses & 57 & $23,4 \%$ & {$[18,2-29,2]$} & 85 & $42,5 \%$ & {$[32,0 \%-53,0 \%]$} & 63 & $53 \%$ & {$[40,7 \% 65,3-\%]$} \\
\hline \multicolumn{10}{|l|}{ Hemoglobina } \\
\hline$\leq 9,0 \mathrm{~g} \%$ & 65 & $26,6 \%$ & {$[21,2-32,7]$} & 71 & $35,5 \%$ & {$[24,4 \%-46,6 \%]$} & 24 & $21,3 \%$ & {$[4,9 \%-37,7 \%]$} \\
\hline 9,1 a $13,0 \mathrm{~g} \%$ & 161 & $66,0 \%$ & {$[59,7-71,9]$} & 110 & $55,0 \%$ & {$[45,7 \%-64,3 \%]$} & 77 & $68 \%$ & {$[57,6 \%-78,4 \%]$} \\
\hline$\geq 13,1 \mathrm{~g} \%$ & 16 & $6,6 \%$ & {$[3,8-10,4]$} & 19 & $9,5 \%$ & {$[-3,7 \%-22,7 \%]$} & 12 & $10,7 \%$ & {$[-6,8 \%-28,2 \%]$} \\
\hline \multicolumn{10}{|l|}{ KT/V } \\
\hline$\leq 0,9$ & 17 & $7,0 \%$ & {$[4,1-10,9]$} & 12 & $6,0 \%$ & {$[-7,4 \%-19,4 \%]$} & 6 & $5 \%$ & {$[-12,4 \%-22,4 \%]$} \\
\hline 0,91 a 1,2 & 70 & $28,7 \%$ & {$[23,1-34,8]$} & 37 & $18,5 \%$ & {$[6,0 \%-31,0 \%]$} & 27 & $24 \%$ & {$[7,9 \%-40,1 \%]$} \\
\hline$\geq 1,21$ & 152 & $62,3 \%$ & {$[58,9-68,4]$} & 151 & $75,5 \%$ & {$[68,6 \%-82,4 \%]$} & 80 & $71 \%$ & {$[61,1 \%-80,9 \%]$} \\
\hline Total & 244 & $100 \%$ & & 200 & $100 \%$ & & 113 & $100 \%$ & \\
\hline
\end{tabular}

As variáveis sociodemográficas e clínicas dos pacientes com risco de suicídio estão representadas na Tabela 2. O gênero feminino esteve associado à presença do transtorno e a medida desta associação demonstrou risco aumentado e significante nas duas etapas iniciais $(\mathrm{OR}=2,77$ na

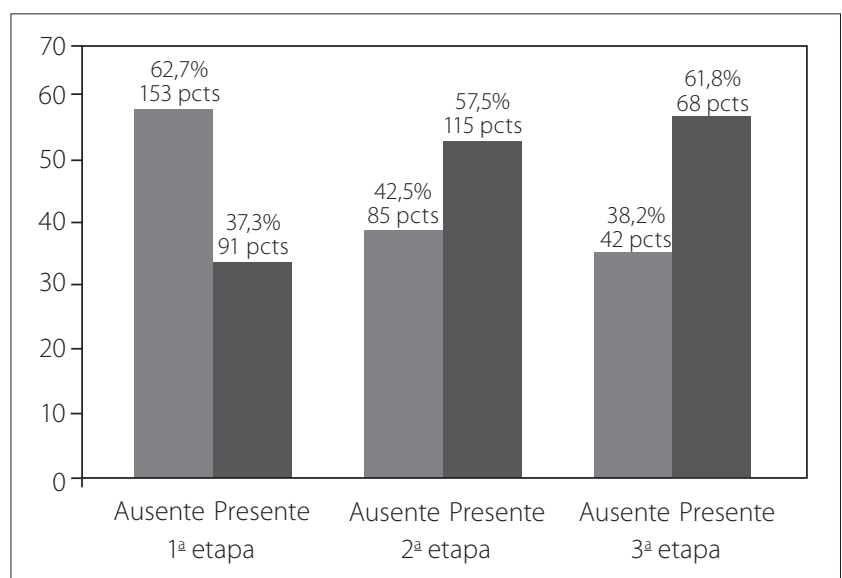

Gráfico 1. Prevalência dos transtornos psiquiátricos encontrados quando considerados em conjunto, nas três etapas primeira, 2,75 na segunda e 1,75 na terceira etapa). Pacientes viúvos, separados e solteiros foram as outras variáveis associadas e com risco elevado da ocorrência da condição. Idade, tempo em diálise, anemia e KT/V (índice fracional de remoção de uréia) baixo não estiveram associados.

Da primeira para a segunda etapa, os $40(16,4 \%)$ pacientes com risco de suicídio evoluíram do seguinte modo: nove deles foram a óbito e 29 continuaram a apresentar o quadro. Dois pacientes evoluíram para fobia social e ansiedade; 20 outros pacientes da amostra se somaram aos 29 que continuavam com a condição, totalizando 49, por ocasião da segunda etapa.

$\mathrm{Na}$ evolução da segunda para a terceira etapa, dos 49 pacientes $(24,5 \%)$ com risco de suicídio, 13 deles foram a óbito antes do final do estudo e sete continuaram a apresentar o quadro. Os outros 29 pacientes restantes evoluíram para ansiedade (5), episódio depressivo maior (3) e fobia social (3), e 18 pacientes foram transferidos de unidade antes da etapa final do estudo. O Gráfico 2 representa os números absolutos e os percentuais do risco de suicídio em relação à amostra analisada. 
Tabela 2. Variáveis sociodemográficas dos pacientes com risco de suicídio nas três etapas do estudo

\begin{tabular}{|c|c|c|c|c|c|c|c|c|c|}
\hline \multirow[t]{2}{*}{ Variáveis } & \multicolumn{3}{|c|}{ 1 Etapa } & \multicolumn{3}{|c|}{$2^{\mathrm{a}}$ Etapa } & \multicolumn{3}{|c|}{ 3a Etapa } \\
\hline & $n$ & $\%$ & IC $95 \%$ & $n$ & $\%$ & IC $95 \%$ & $n$ & $\%$ & IC $95 \%$ \\
\hline \multicolumn{10}{|l|}{ Demográficas } \\
\hline \multicolumn{10}{|l|}{ Sexo } \\
\hline Masculino & 12 & $30 \%$ & {$[24,1-35,8]$} & 15 & $30,6 \%$ & {$[24,1-35,8]$} & 2 & $28,5 \%$ & {$[12,4-46,1]$} \\
\hline Feminino & 28 & $70 \%$ & {$[65,2-77,9]$} & 34 & $69,4 \%$ & {$[65,0-77,6]$} & 5 & $71,5 \%$ & {$[59,2-86,9]$} \\
\hline \multicolumn{10}{|l|}{ Faixa etária } \\
\hline$\leq 20$ anos & 2 & $5 \%$ & {$[0,6-8,8]$} & 3 & $6,0 \%$ & {$[-8,2-13,3]$} & 0 & $0 \%$ & \\
\hline 21 a 40 anos & 16 & $40 \%$ & {$[30,6-48,2]$} & 20 & $40,7 \%$ & {$[31,6-48,8]$} & 1 & $14,2 \%$ & {$[5,7-25,3]$} \\
\hline 41 a 60 anos & 14 & $35 \%$ & {$[28,6-39,9]$} & 17 & $34,7 \%$ & {$[29,1-40,3]$} & 2 & $28,5 \%$ & {$[12,4-46,1]$} \\
\hline$\geq 61$ anos & 8 & $20 \%$ & {$[15,5-25,2]$} & 9 & $18,3 \%$ & {$[13,6-25,4]$} & 4 & $57,1 \%$ & {$[21,5-82,5]$} \\
\hline \multicolumn{10}{|l|}{ Estado civil } \\
\hline Casado & 15 & $37,5 \%$ & {$[33,2-43,1]$} & 17 & $34,7 \%$ & {$[30,0-38,2]$} & 2 & $28,5 \%$ & {$[12,4-46,1]$} \\
\hline Solteiro & 19 & $47,5 \%$ & {$[39,9-52,4]$} & 23 & $46,9 \%$ & {$[40,5-53,1]$} & 3 & $42,8 \%$ & {$[21,4-57,5]$} \\
\hline Viúvo & 3 & $7,5 \%$ & {$[4,5-9,8]$} & 4 & $8,1 \%$ & {$[-5,6-14,1]$} & 2 & $28,5 \%$ & {$[12,4-46,1]$} \\
\hline Separado & 3 & $7,5 \%$ & {$[4,6-9,8]$} & 5 & $10,2 \%$ & {$[-4,4-16,9]$} & 0 & $0 \%$ & \\
\hline \multicolumn{10}{|l|}{ Clínicas } \\
\hline \multicolumn{10}{|l|}{ Tempo em diálise } \\
\hline$\leq 12$ meses & 7 & $17,5 \%$ & {$[14,4-22,5]$} & 0 & $0 \%$ & & 0 & $0 \%$ & \\
\hline 13 a 24 meses & 9 & $22,5 \%$ & {$[17,2-26,8]$} & 7 & $14,2 \%$ & {$[-1,2-23,6]$} & 0 & $0 \%$ & \\
\hline 25 a 36 meses & 4 & $10 \%$ & {$[7,2-15,4]$} & 8 & $16,3 \%$ & {$[2,5-25,2]$} & 0 & $0 \%$ & \\
\hline 37 a 48 meses & 4 & $10 \%$ & {$[7,2-15,4]$} & 9 & $18,3 \%$ & {$[4,6-29,2]$} & 1 & $14,2 \%$ & {$[5,7-25,3]$} \\
\hline 49 a 60 meses & 5 & $12,5 \%$ & {$[7,9-16,7]$} & 13 & $26,5 \%$ & {$[4,8-37,3]$} & 2 & $28,5 \%$ & {$[12,4-46,1]$} \\
\hline$\geq 61$ meses & 11 & $27,5 \%$ & {$[22,2-31,4]$} & 12 & $24,4 \%$ & {$[12,0-33,0]$} & 4 & $57,1 \%$ & {$[21,5-82,5]$} \\
\hline \multicolumn{10}{|l|}{ Hemoglobina } \\
\hline$\leq 9,0 \mathrm{~g} \%$ & 8 & $20 \%$ & {$[15,2-25,2]$} & 9 & $18,3 \%$ & {$[10,5-40,6]$} & 2 & $28,5 \%$ & {$[12,4-46,1]$} \\
\hline 9,1 a $13,0 \mathrm{~g} \%$ & 26 & $65 \%$ & {$[59,2-68,8]$} & 30 & $61,2 \%$ & {$[50,1-68,7]$} & 4 & $57,1 \%$ & {$[21,5-82,5]$} \\
\hline$\geq 13,1 \mathrm{~g} \%$ & 6 & $15 \%$ & {$[12,7-18,4]$} & 10 & $20,4 \%$ & {$[7,7-29,7]$} & 1 & $14,2 \%$ & {$[5,7-25,3]$} \\
\hline \multicolumn{10}{|l|}{$\mathrm{KT} / \mathrm{V}$} \\
\hline$\leq 0,9$ & 3 & $7,5 \%$ & {$[4,5-9,8]$} & 5 & $10,2 \%$ & {$[-2,4-17,8]$} & 1 & $14,2 \%$ & {$[5,7-25,2]$} \\
\hline 0,91 a 1,2 & 12 & $30 \%$ & {$[24,1-35,8]$} & 16 & $32,6 \%$ & {$[16,6-41,0]$} & 3 & $42,8 \%$ & {$[21,4-57,5]$} \\
\hline$\geq 1,21$ & 25 & $62,5 \%$ & {$[58,9-67,5]$} & 28 & $57,1 \%$ & {$[48,6-72,7]$} & 4 & $57,1 \%$ & {$[21,5-82,5]$} \\
\hline Total & 40 & $100 \%$ & & 49 & $100 \%$ & & 7 & $100 \%$ & \\
\hline
\end{tabular}

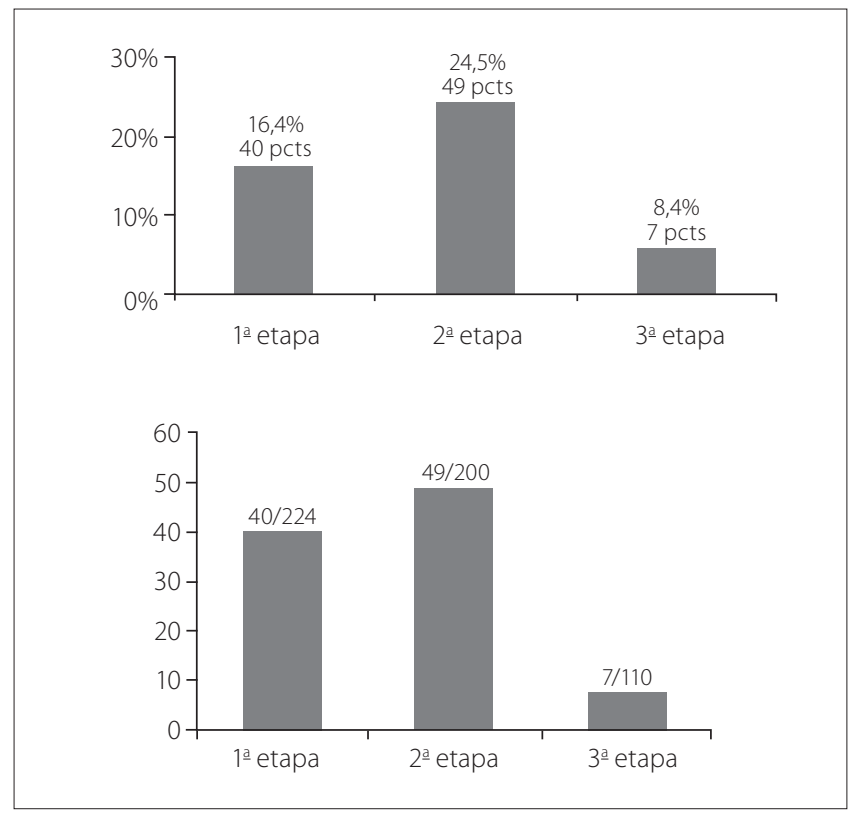

Gráfico 2. Variação do risco de suicídio em números percentuais e absolutos nas três etapas do estudo
A mortalidade estudada foi a do início do estudo até o seu final, depois de três anos. Foi utilizada a curva de Kaplan-Meier para analisar a sobrevida do total da amostra. Ela também foi aplicada para comparar a sobrevida entre os 40 pacientes que apresentavam risco de suicídio por ocasião da primeira etapa e aqueles 153 que não apresentaram nenhum transtorno no início do estudo (Log Rank Test). As curvas estão representadas pelos Gráficos 3 e 4.

Da amostra inicial de 244 pacientes que foram estudados pelo questionário na primeira etapa, 32 morreram antes da segunda etapa e 29 antes da terceira e última etapa, totalizando 61 óbitos. Dos 40 pacientes $(16,4 \%)$ com risco de suicídio, nove deles foram a óbito antes da segunda etapa e 13 antes do final do estudo. O total de óbitos foi de 22 pacientes. Dos 153 pacientes que não apresentavam nenhum transtorno psiquiátrico à época, o número de pacientes que faleceram também foi de 22 (14,4\%), quando o esperado era de $38,6(p=0,000)$.

A regressão de Cox foi utilizada em razão da necessidade de censurar algumas variáveis independentes que se 


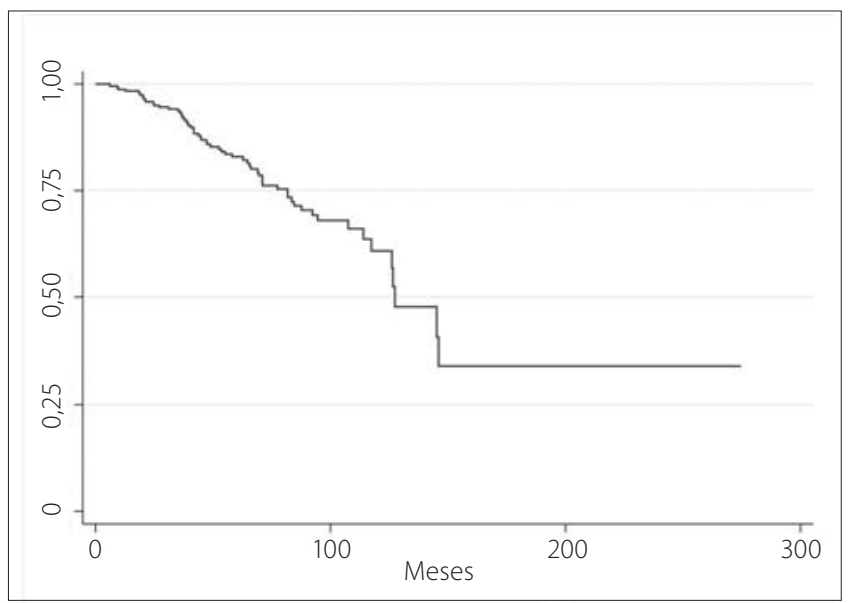

Gráfico 3. Curva de Kaplan-Meier da sobrevida global da amostra no decorrer dos três anos do estudo

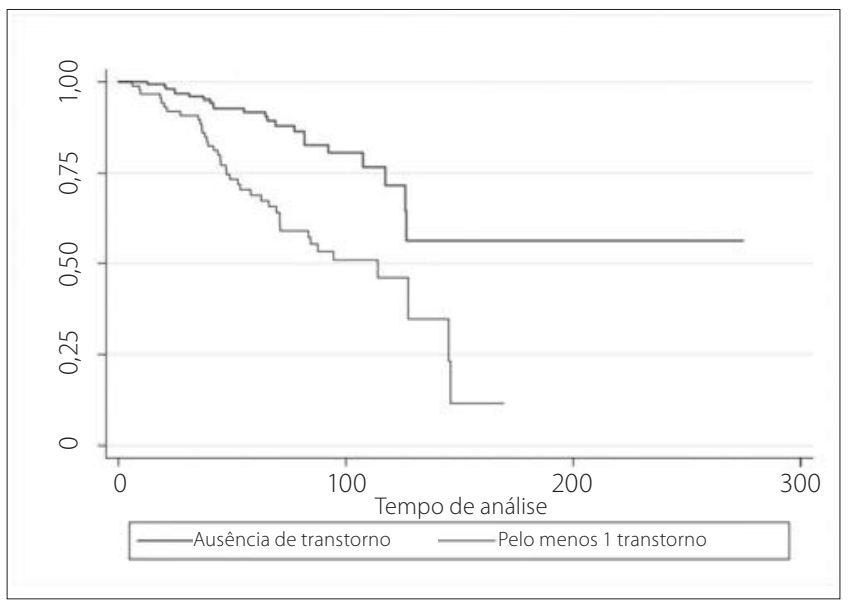

Gráfico 4. Curva de Kaplan-Meier comparativa da sobrevida dos dois grupos de pacientes da amostra (com e sem risco de suicídio) - log rank test

modificavam em função do tempo, como pacientes transferidos, tempo em diálise, recusa em responder ao questionário e tempo final de tratamento. Todas as variáveis sociodemográficas e clínicas do estudo foram analisadas como causas de morte, de maneira bi e multivariadas, e não apresentaram aumento de risco relativo estatisticamente significante.

A incidência de óbitos quando se considerou a amostra como um todo foi de 4,4 pacientes por 100 por ano. A incidência de óbitos nos pacientes sem o transtorno foi de 3,35 por 100 pacientes por ano, enquanto naqueles pacientes que apresentavam risco de suicídio, a taxa de incidência foi de 9,91 pacientes por 100 por ano. O RR pelo modelo de Cox para estes pacientes foi de 2,87; IC 95\% [1,69-4,87].

\section{DISCUSSÃO}

O presente trabalho pretendeu preencher uma lacuna, uma vez que são raros ou inexistentes os estudos que avaliam o risco de suicídio em pacientes em programa de diálise no Brasil. A maioria dos trabalhos publicados prioriza os transtornos afetivos, como depressão e ansiedade, deixando de lado a abordagem de outros transtornos ${ }^{4,8,10}$.

As dificuldades psicossociais apresentadas pelos pacientes em programa crônico de diálise têm sido alvo de interesse crescente nas últimas décadas e os aspectos psiquiátricos adquirem grande importância no diagnóstico e nas estratégias de tratamento destes pacientes. Considerada uma complicação da depressão, o risco de suicídio encontra-se associado fortemente ao prognóstico e à sobrevida desta população ${ }^{10-12}$.

É especialmente preocupante a quantidade de pacientes com risco de suicídio que foram diagnosticados neste nosso trabalho, principalmente nas fases iniciais. Foram 40 pacientes na primeira etapa, 49 na segunda e sete na terceira etapa.

As estimativas de suicídio nessa população podem variar. Abram, Moore e Westervelt11 avaliaram estes números, envolvendo 127 centros de diálise, aos quais enviou questionários, obtendo dados de 3.478 pacientes. De acordo com os dados coletados, aproximadamente 5\% dos pacientes levou a cabo a intenção de cometer suicídio. Segundo os autores, o fator crucial que influencia alguns pacientes a escolherem a descontinuidade do tratamento é a sua própria percepção do prognóstico da doença.

A mortalidade no nosso estudo se comprovou alta, uma vez que 22 pacientes com este transtorno foram a óbito antes do final. A regressão de Cox que estimou o risco relativo daqueles com tendência suicida encontrou risco significante de quase três vezes.

A ideação suicida relaciona-se com a mortalidade em pacientes com IRC em todas as suas fases, seja na diálise, seja no pós-transplante. É extremamente importante o seu reconhecimento, mesmo porque se trata de condição passível de tratamento, seja pela psicoterapia ou pela terapia farmacológica.

Esse alto índice de mortalidade nos pacientes que apresentavam diagnóstico dessa condição no início do estudo confirma numerosos relatos da literatura que são unânimes em colocar transtornos psiquiátricos, principalmente os afetivos, como contribuintes importantes na sobrevida de pacientes renais crônicos em terapia dialítica ${ }^{23-25}$.

Não nos foi possível determinar as causas das mortes desses pacientes e esse fato constitui uma possível limitação, uma vez que a análise destas causas poderia esclarecer se aqueles pacientes com idéias suicidas tiveram êxito na sua intenção, ou não. 
Nas últimas décadas, a produção científica acerca do suicídio revestiu-se de caráter predominantemente pragmático e técnico. Nessa linha predominam os estudos que buscam identificar fatores de risco, recortando o evento em múltiplas variáveis em nível biológico, psicológico e social. Aparentemente, essas pesquisas não têm sido capazes de reverter a tendência de aumento do suicídio, observada em vários países.

Uma das definições de suicídio refere-se ao ato humano de causar a cessação da própria vida. Essa definição implica, em primeiro lugar, que o termo suicídio só pode ser usado no caso de morte ou de circunstâncias cuja seqüência causal leve à morte e na qual tenha havido intencionalidade do sujeito. Como anteriormente referido, essa pretensa intenção ao ato não foi possível detectar neste nosso estudo ${ }^{26}$.

Consideram-se fatores associados ao suicídio: as tentativas prévias, a doença afetiva, o isolamento social, a história familiar, a declaração de intenções e uma série de variáveis demográficas e socioeconômicas acrescentam-se as situações de eventos estressores e doença terminal. Quatro situações são descritas como as mais importantes no comportamento suicida: o aumento na prevalência de transtornos depressivos, as mudanças psicobiológicas, o aumento no número de estressores sociais e a mudança nos padrões de aceitação de comportamentos suicidas e aumento na disponibilidade de modelos suicidas ${ }^{20}$. Praticamente todos esses dados estão presentes na situação dos nossos pacientes em diálise, defrontados que estão com essa problemática na sua extensão.

Como entrevista, o MINI se confirmou de fácil e rápida execução, tendo os pacientes relatado boa compreensão de todos os módulos, especialmente o referente ao risco de suicídio, com perguntas que exigem apenas respostas diretas, não havendo referências a dificuldades. Entretanto, deve-se considerar a possibilidade de diagnósticos falso-negativos, fato que certamente possibilitará maior atenção da equipe médica com a intenção de mais bem esclarecer o diagnóstico, talvez reparando a subavaliação descrita desta condição.

A comparação dos resultados deste trabalho com outros estudos deve levar em consideração a inexistência de estudos que tenham utilizado metodologia similar, assim como, considerar a existência nesses pacientes de uma constelação de sintomas que possam alterar os critérios diagnósticos dos transtornos psiquiátricos. Torna-se crucial a existência de estudos sistemáticos de transtornos psiquiátricos neste subgrupo de pacientes que utilizem metodologias semelhantes, como já ocorrem em portadores de outras doenças crônicas, como as neoplasias²7.

Finalmente, é possível considerar que a nossa amostra foi representativa dos pacientes em programa de diálise na Bahia, os quais também exibem características sociodemográficas e clínicas similares às de pacientes em outros centros do Brasil. Entretanto, comparações da nossa amostra com populações de fora do País são mais problemáticas, por estas apresentarem perfil epidemiológico distinto. A generalização dos nossos achados depende, em última análise, de similaridades existentes entre diversas amostras.

Esperamos que os dados e os resultados descritos possam ser úteis para estimular outras pesquisas nesta área, e que venham servir de alerta para a alta prevalência e a elevada mortalidade dos pacientes portadores de tendência suicida no âmbito das unidades de diálise. Finalmente, que possam contribuir, de alguma forma, na melhora da abordagem e no aumento da sobrevida dos pacientes portadores de IRC em programa de hemodiálise.

\section{CONCLUSÕES}

O risco de suicídio foi uma condição psiquiátrica prevalente e associada com o aumento da mortalidade em pacientes em programa de diálise. O risco relativo de morte daqueles com este transtorno psiquiátrico foi de quase três vezes.

\section{REFERÊNCIAS}

1. Dialysis Statistics. Available from: URL: http://www.globoldialysis.com/stats.asp.

2. Sociedade Brasileira de Nefrologia. Censo de 2006. SBN; 2006.

3. Comissão Estadual de Nefrologia. Secretaria Estadual de Saúde. Suraps. Relatório Anual. 1999. p.1-12.

4. Nichols KA, Springford V. The psycho-social stressors associated with survival by dialysis. Behav Res Ther. 1984;22(5):563-74.

5. D'Amico G. Comparability of the different registries on renal replacement therapy. Am J Kidney Dis. 1995;25(1):113-8.

6. Lloyd GG. Textbook of general hospital psychiatry. Edinburg: Churchill Livingstone; 1991.

7. Cohen LM. Renal disease. In: Rundell JR, Wise MG, editores. Textbook of consultationliaison psychiatry. Washington (DC): American Psychiatric Press; 1996.

8. Kutner NG, Fair PL, Kutner MH. Assessing depression and anxiety in chronic dialysis patients. J Psychosom Res. 1985;29(1):23-31.

9. Kimmel PL, Thamer M, Richard CM, Ray NF. Psychiatric illness in patients with end-stage renal disease. Am J Med. 1998;105(3):214-21.

10. Burton $H$, Kline $S$, Lindsay R. The relationship of depression to survival in chronic renal failure. Psychosomatic Medicine. 1986;48:261-9.

11. Abram HS, Moore GL, Westervelt FB. Suicidal behavior in chronic dialysis patients. Am J Psychiatry. 1971;127(9):1199-204.

12. Almeida MA, Meleiro AM. Depressão e insuficiência renal crônica: uma revisão. J Bras Nefrol. 2000;22(1):21-9.

13. Desjarlais R, Eisenberg L, Good B, Kleinman A. Salud mental en el mundo: problemas y prioridades en poblaciones de bajos ingresos. Organización Panamericana de la Salud, 1997.

14. Engberg M. Mortality and suicide rates of involuntarily comitted patient. Acta Psychiatr Scand. 1994;89:35-40.

15. Banco Mundial. Informe sobre el desarrollo mundial 1993: invertir em salud. Oxford New York: University Press; 1993.

16. Diekstra, RFW. The epidemiology of suicide and parasuicide. Acta Psychiatr Scand. 1993:371:9-20.

17. Fundação Nacional de Saúde. Mortalidade - Brasil - 1995. Brasília: CENEPI/Ministério da Saúde; 1998. 
18. Mello Jorge MHP. Mortalidade por causas violentas no Município de São Paulo [tese]. São Paulo: Faculdade de Saúde Pública da Universidade de São Paulo, 1995.

19. Kennedy SH, Craven JL, Rodin GM, Roin GM. Major depression in renal dialysis patients: an open trial of antidepressant therapy. J Clin Psychiatry. 1989;50(2):60-3.

20. Rogers JR. Theoretical grounding: 'the missing link' in suicide research. J Counsel Dev. 2001;79:16-29.

21. Sheehan DV, Lecrubier Y, Sheehan KH, Amorim P, Janavs J, Weiller E, et al. The MiniInternational Neuropsychiatric Interview (M.I.N.I.): the development and validation of a structured diagnostic psychiatric interview for DSM-IV and ICD-10. J Clin Psychiatry. 1998;59(Suppl 20):22-33.

22. Moura Jr. JA, Marcilio de Souza CM, Oliveira IR. Prevalência de transtornos psiquiátricos em pacientes em hemodiálise no estado da Bahia. J Bras Psiquiatria. 2006;55(3):178-83.
23. McClellan WM, Anson C, Birkeli K, Tuttle E. Functional status and quality of life: predictors of early mortality among patients entering treatment for end stage renal disease. J Clin Epidemiol. 1991;44(1):83-9.

24. Peterson RA, Kimmel PL, Sacks CR, Mesquita ML, Simmens SJ, Reiss D. Depression, perception of illness and mortality in patients with end-stage renal disease. Int J Psychiatry Med. 1991;21(4):343-54

25. Wai L, Richmond J, Burton H, Lindsay RM. Influence of psychosocial factors on survival of home-dialysis patients. Lancet. 1981;2(8256):1155-6.

26. Krug EG, organizador. Informe mundial sobre la violencia y la salud. Washington (DC): OPS/OMS; 2003

27. Kathol RG, Mutgi A, Williams J, Clamon G, Noyes R. Diagnosis of major depression in cancer patients according to four sets of criteria. Am J Psychiatry. 1990;147(8):1021-4. 